\title{
Evaluation of the EC8-3 confidence factors for the characterization of concrete strength in existing structures
}

\author{
Xavier Romão • Rui Gonçalves • Aníbal Costa • \\ Raimundo Delgado
}

Received: 16 January 2012/ Accepted: 7 May 2012

(C) RILEM 2012

\begin{abstract}
A probabilistic framework is defined to evaluate the values of the Confidence Factors (CFs) proposed in Eurocode 8 Part 3 (EC8-3) for the characterization of material properties. This evaluation is presented for the concrete compressive strength but its validity for other material properties can also be inferred from the results obtained. The number of material tests and the existence of prior knowledge are the essential aspects for the CF quantification. The probabilistic framework proposed in the first part of the study does not consider the existence of prior knowledge and is based on the concept of confidence intervals. In the second part of the study, the effects of prior knowledge are considered using a Bayesian framework. The combination of testing data obtained from different types of tests is also addressed as an extension of the referred Bayesian approach. Results indicate that the EC8-3 proposed CFs for KL1 and KL2 are adequate, but for KL3 it is suggested that a larger value should be used.
\end{abstract}

X. Romão $(\bowtie) \cdot$ R. Gonçalves · R. Delgado

Civil Engineering Department, Faculdade de Engenharia da Universidade do Porto, Rua Dr. Roberto Frias, 4200-465 Porto, Portugal

e-mail: xnr@fe.up.pt

A. Costa

Civil Engineering Department, Universidade de Aveiro, Campus Universitário de Santiago, 3810-193 Aveiro, Portugal
Keywords Confidence Factor - Eurocode 8 Part 3 . Existing structures $\cdot$ Safety assessment $\cdot R C$ structures

\section{Introduction}

There are numerous differences between the design of a new structure according to structural design codes and the assessment of the same structure after many years in service [1]. Although the properties of an existing structure can be known to a certain extent, it should be noted that a number of uncertainties may arise from on-site inspection/testing and other procedures carried out to check the actual condition of the structure. For example, techniques for in situ testing may be considered to be reasonably well developed but they involve measurement errors and the interpretation of the results may imply a considerable degree of uncertainty [2]. The consideration of several expert opinions on a specific matter is another possible source of uncertainty since opinions can vary considerably depending on the complexity of the problem at hand [3]. In cases where important data have been lost, the need to estimate data values based on past experience or on the existence of similar structures also increases the uncertainty about the knowledge of the existing construction.

With respect to the assessment of the material properties of existing structures, these can be obtained with varying degrees of accuracy based on in situ measurements. In order to deal with the uncertainty of 
those measurements, different degrees of knowledge are established by the codes, which reflect the type and quality of the gathered data, [4-6]. To reflect the referred levels of knowledge in a quantitative manner and to account for them in the assessment, penalty factors can be associated to those levels that will either reduce the "capacity" or increase the "demand". In this context, the evaluation of the referred penalty factors, which are termed Confidence Factors (CF), is addressed herein following the definition proposed in EC8-3 [4]. Although the values of the CFs to be used in a given country can be found in its National Annex, recommended values of the CFs are also proposed within the EC8-3 main document. Therefore, the present study assesses the reliability of those recommended values by establishing a probabilistic framework for their evaluation. Even though the general concept behind the consideration of CFs is independent of the type of structural material, their evaluation is presented herein for the case of reinforced concrete (RC) structures. Nonetheless, given the format of the proposed probabilistic approach, results for other material properties can also be inferred.

The proposed study contains two parts. The first one, presented in Sect. 3, addresses the major part of the probabilistic framework that is developed to analyse the reliability of the CFs proposed by EC83. This first part is developed in light of several conditions established in Sect. 2 that are based on the interpretation of the EC8-3 procedures for seismic safety assessment and on existing research on this topic. The second part of the study, which is presented in Sect. 4, addresses the situation of combining different sources of information for the purpose of assessing the material properties. The general outline of the necessary Bayesian framework is analysed and the combination of different sources of information are addressed within the scope of the EC8-3 procedures.

\section{General framework for the definition of the CF}

\subsection{The CF in the EC8-3 procedures for seismic safety assessment}

In EC8-3, the previously referred Knowledge Level (KL) is defined by combining the knowledge available in the following items: geometry, details and materials. In the context of RC structures, geometry refers to the geometrical identification of the structural elements, details refers to the amount and detailing of the reinforcement, and materials refers to the mechanical properties of the steel and concrete. Knowledge on the geometry is provided either by the original construction drawings or by survey. Details and materials are obtained through inspection and testing, respectively. EC8-3 defines three levels of knowledge, denoted by KL1, KL2 and KL3 in increasing order of comprehensiveness, and also defines a CF associated with each level. The recommended values of these factors are 1.35, 1.2 and 1.0, for KL1, KL2 and KL3, respectively, and Table 1 summarises the combinations of information defining the KLs. The terms visual, full, limited, extended and comprehensive are defined in the code together with the recommended minimum amount of operations related to survey, inspection and testing. For the case of assessing

Table 1 General definition of the knowledge levels

\begin{tabular}{|c|c|c|c|c|}
\hline $\begin{array}{l}\text { Knowledge } \\
\text { level }\end{array}$ & Geometry & Details & Materials & $\mathrm{CF}$ \\
\hline KL1 & \multirow{3}{*}{$\begin{array}{l}\text { From original architectural } \\
\text { drawings with sample } \\
\text { visual survey or from full } \\
\text { survey }\end{array}$} & $\begin{array}{l}\text { Simulated design in accordance with } \\
\text { relevant practice and from limited in-situ } \\
\text { inspection }\end{array}$ & $\begin{array}{l}\text { Default values according to } \\
\text { standards of the time of } \\
\text { construction and from limited } \\
\text { in-situ testing }\end{array}$ & 1.35 \\
\hline KL2 & & $\begin{array}{l}\text { From incomplete original detailed } \\
\text { construction drawings with limited in } \\
\text { situ inspection or from extended in situ } \\
\text { inspection }\end{array}$ & $\begin{array}{l}\text { From original design } \\
\text { specifications with limited in } \\
\text { situ testing or from extended in- } \\
\text { situ testing }\end{array}$ & 1.20 \\
\hline KL3 & & $\begin{array}{l}\text { From original detailed construction } \\
\text { drawings with limited in situ inspection } \\
\text { or from comprehensive in-situ inspection }\end{array}$ & $\begin{array}{l}\text { From original test reports with } \\
\text { limited in situ testing or from } \\
\text { comprehensive in-situ testing }\end{array}$ & 1.00 \\
\hline
\end{tabular}


Table 2 Recommended minimum percentage of elements to check for details and minimum number of tests of material samples, per floor and type of primary element for different KLs

\begin{tabular}{llll}
\hline $\begin{array}{l}\text { Knowledge } \\
\text { level }\end{array}$ & Level of testing & $\begin{array}{l}\text { Percentage of } \\
\text { elements to } \\
\text { check for details }\end{array}$ & $\begin{array}{l}\text { Number of } \\
\text { material } \\
\text { samples }\end{array}$ \\
\hline KL1 & Limited & 20 & 1 \\
KL2 & Extended & 50 & 2 \\
KL3 & Comprehensive & 80 & 3 \\
\hline
\end{tabular}

material strengths, which is the context of the present study, the terms limited, extended and comprehensive are quantified in Table 2 and correspond to the minimum number of material samples per floor and type of primary element (beam, column or wall) that are needed to assess the material strength according to a chosen KL.

From the safety assessment stage point of view, depending on the type of analysis method that is selected (linear or nonlinear) and on the type of mechanism to be checked (ductile or brittle), the capacity is usually reduced by the CF. On the other hand, situations will occur where the demand needs to be increased by the $\mathrm{CF}$, namely if certain conditions are met when examining brittle mechanisms based on linear analysis [4]. The capacity reduction case is the focus of the present study for which two different situations are defined in EC8-3. With respect to the safety assessment of a certain ductile mechanism, its capacity is obtained from a given expression where the material strength values are considered with mean values divided by the $C F$. In the case of a brittle mechanism of a primary element, EC8-3 sets a larger safety margin as the capacity is obtained from a given expression where the material strength values are considered with mean values divided by the CF and by the partial safety factor of the corresponding material. Of the two situations, the former is considered to be more critical.

Although the EC8-3 procedures acknowledge the importance of the existing KL about the geometry, the details and the materials, their interpretation leads to conclude that the definition of the CFs does not depend on all of these items. Namely, it can be seen, by analysing Table 1, that knowledge requirements related to the geometry are the same for all KLs. This implies that the existing information about the geometry is expected to be reliable enough to allow the definition of an adequate numerical model of the structure, irrespective of the selected KL. Therefore, it is concluded that, according to the EC8-3 procedures, the $\mathrm{CF}$ values do not depend on the uncertainty that may exist about the geometry. With respect to the details, Tables 1 and 2 indicate that selecting a KL requires that a certain amount of knowledge must be obtained about parameters such as longitudinal and transversal reinforcement ratios, and stirrup spacing (for the particular case of reinforced concrete structures). Bearing in mind that, in many cases, an adequate knowledge is only obtained if extensive and intrusive methods are considered, the use of such methods must be weighted against the implications on the continuous use of the building during these surveys. Considering that situations for which knowledge about the details of certain members is not available will occur, the analyst will have to decide whether to characterize them by using information on similar members or by using conservative estimates. In any case, the EC8-3 procedures are unclear regarding the practical effects of the existing uncertainty about the details since the CF only affects the material properties. To illustrate this situation, Eq. (1) presents the EC8-3 proposed expression for the Near Collapse chord rotation capacity $\theta_{\mathrm{NC}}$, where $\gamma_{e l}$ is 1.5 for primary members and 1.0 for secondary ones, $v$ is the normalized axial force, $\omega$ and $\omega^{\prime}$ are the mechanical reinforcement ratios of the tension and compression, respectively, longitudinal reinforcement, $f_{y w}$ is the stirrup yield strength, $\rho_{s x}$ is the ratio of transverse steel area $A_{s x}$ parallel to the direction of loading, $\rho_{d}$ is the steel ratio of diagonal reinforcement (if any) in each diagonal direction and $\alpha$ is the confinement effectiveness factor [4]:

$$
\begin{aligned}
\theta_{\mathrm{NC}}= & \frac{1}{\gamma_{e l}} \cdot 0.016 \cdot 0.3^{v} \cdot\left(\frac{\max \left(0.01, \omega^{\prime}\right)}{\max (0.01, \omega)}\left(\frac{f_{c}}{\mathrm{CF}}\right)\right)^{0.2} \\
& \cdot\left(\frac{L_{s}}{h}\right)^{0.35} \cdot 25^{\alpha \cdot \rho_{s x} \cdot \frac{\left(f_{y w} / \mathrm{CF}\right)}{\left(c_{c} / \mathrm{CF}\right)} \cdot 1.25^{100 \rho_{d}}}
\end{aligned}
$$

As can be seen, only the terms $f_{y w}$ and $f_{c}$ are divided by the CF while the terms $\omega, \omega^{\prime}, \rho_{s x}$ and $\rho_{d}$ related to the details remain unaffected, meaning that uncertainty is only explicitly reflected on the values of the material properties. Since knowledge about the details does not imply an increase of the knowledge about the 
material properties (and vice versa), the two aspects are seen to be independent and it can be argued that the format of Eq. (1) reflects an indirect influence of the uncertainty related to the details that is inadequate in some cases. For example, in a situation where the knowledge about the material properties is obtained according to KL3 but the knowledge about the details can only be obtained according to KL1, the considered $\mathrm{CF}$ should be that of KL1. Since this CF value is overconservative for the material properties, as demonstrated in the following sections, and since, as stated before, it does not affect the detail-related properties, its consideration in the present EC8-3 format does not reflect adequately the true uncertainty.

Given the previous interpretation of the EC8-3 procedures, as well as arguments resulting from previous research addressing the adequacy of the $\mathrm{CF}$ concept to account for several types of uncertainties entering the seismic safety assessment process [7-12], the proposed study only focuses the evaluation of the $\mathrm{CF}$ values in the context of the characterization of the material properties. This is equivalent to say that $\mathrm{CF}$ values are evaluated assuming that KL3 conditions are available for the characterization of the details. In the opinion of the authors, the adequate consideration of the uncertainty about the details requires a modification of the current EC8-3 format for the capacity expressions by introducing, for example, corrective factors affecting the detail-related parameters. Furthermore, as noted in [11], the influence of the detailrelated uncertainty should also be reflected on the demand side of the assessment due to its potential influence on the global behaviour of the structure. However, such in-depth assessment of these aspects falls outside the scope of the present study.

\subsection{Quantification of the CFs}

According to EC8-3, when there is no prior knowledge about the materials under assessment, the $\mathrm{CF}$ values depend mainly on the number of tests that are performed to assess the material properties of interest, hereafter simply referred as strength values. When there is prior knowledge (e.g. based on available design data or previous testing data), EC8-3 reflects the consideration of this information by reducing the required number of tests of a given KL. For the purpose of the following analysis regarding the minimum number of required material tests and of the subsequent probabilistic quantification of the CFs presented in Sect. 3, it is assumed there is no prior knowledge about the materials under assessment. The situation of existing prior knowledge is addressed in Sect. 4.

Considering the situation with no prior knowledge, the following two scenarios are analysed to establish the worst case scenario, i.e. that corresponding to larger uncertainty, which will serve as the basis of the probabilistic framework:

Scenario 1-Only destructive tests are used to characterize the material properties;

Scenario 2-Different types of tests are combined to characterize the material properties, i.e. destructive tests and non-destructive tests (NDTs) [13-15].

Scenario 2 is more likely to occur in real situations since the cost of NDTs is usually much lower than that of destructive tests. Nonetheless, NDTs have several disadvantages. For example, a reliable functional relation must be established between the property measured by the NDT and the material characteristic under assessment in order to be able to rely on their results. Such relation is usually obtained by performing a regression analysis using NDT results and results of destructive tests from the structure under assessment, e.g. see [16]. Hence, NDTs must always be associated to more reliable destructive tests- a fact also acknowledged by EC8-3. Moreover, results obtained from NDTs have a larger variability than results of destructive tests. Besides the inherent variability of the material property under assessment, additional sources of variability, such as that resulting from the repeatability error associated to a certain NDT and that coming from the error associated to the regression relation, must also be considered, e.g. see $[9,16]$. Nonetheless, the negative effect of this larger variability can be overcome since a larger number of tests can be performed, a fact which can increase considerably the confidence in the results. Therefore, it is concluded that Scenario 2 leads to a characterization of the material properties with less uncertainty. Furthermore, since destructive tests must be carried out in both scenarios, Scenario 2 is seen as an extension of Scenario 1. Therefore, from the point of view of the uncertainty of the results, though using a testing procedure generally agreed to be more reliable, e.g. see [17], Scenario 1 is considered to be more critical, since it will usually involve a lower number of tests. Hence, Scenario 1 will be considered as the basis 
of the development of the probabilistic framework presented in Sect. 3. Still, the combination of results coming from different types of tests (Scenario 2) will be addressed in Sect. 4 since it can be considered as a particular case of the situation where prior knowledge exists.

In the absence of prior knowledge and after selecting a given KL, the EC8-3 defines the minimum number of tests by multiplying the constants given in Table 2 by the number of storeys and by the number of primary element types. For example, considering the simple case of assessing the concrete compressive strength of a one-storey RC frame structure with only beams and columns as primary elements and considering that KL1 is the selected $\mathrm{KL}$, the minimum number of tests is two (one in a beam and one in a column). Although not clearly stated in EC8-3, if two different concrete grades are used in this structure, for example one for beams and one for columns, the minimum number of tests can be interpreted as being one for each concrete grade. Following the same reasoning, if the selected KL is KL3, the previously obtained minimum number of tests is now six, for the case of one concrete grade, and three for each concrete grade, for the case of two concrete grades. Although for taller structures the minimum number of tests will be proportionally larger, the fact remains that for shorter structures EC8-3 allows the determination of mean strength estimates based on a single test result, irrespective of the type of material.

For comparison purposes it is referred that, similar to the EC8-3, the Italian code [5] sets equivalent restrictions to define the $\mathrm{CFs}$ and recommends the same minimum number of tests. However, it sets an additional restriction related to the storey area. If this area exceeds $300 \mathrm{~m}^{2}$, the minimum number of tests must be increased. In the context of assessing characteristic values of concrete compressive strength, it is further noted that [17] refers that a minimum number of three core tests must be considered for the assessment of a certain concrete class, irrespective of the size of the structure. Furthermore, even though only two KLs are defined in [6], the procedures proposed to obtain mean strength values are set depending on the material type and on various constraints established for the definition of the minimum number of material tests. The procedure defined in [6] allows the consideration of existing prior knowledge about the material strength under assessment and of the variability of the test results as factors that will influence both the definition of the $\mathrm{CF}$ and of the required number of tests. For the case of concrete compressive strength, when neglecting other constraints based on the number of floors and on the number of element types, and assuming the existence of only one concrete grade for which there is no prior knowledge, the minimum number of tests set by [6] for both KLs is six. Similar to [5], a restriction related to the maximum storey area is also set in [6]. If this area exceeds $930 \mathrm{~m}^{2}$, the minimum number of tests must be increased.

\section{Probabilistic definition of the CFs}

\subsection{Basic hypotheses and definitions of the probabilistic analysis}

In the context of EC8-3, an estimate $\bar{X}$ of the mean value $\mu$ of a given material property must be divided by a CF in order to provide a value of the property with an adequate safety level. Moreover, the CF value is seen to be larger if there is less knowledge about the material. From a safety perspective, the need for the $\mathrm{CF}$ reflects the underlying critical situation in terms of safety that occurs when $\bar{X}$ overestimates $\mu$. Hence, the present study addresses the probabilistic quantification of the CFs that adjust the mean estimate of a material strength in order to provide a reliable value reflecting the KL that is attained in the assessment.

The number of material tests and the existence of prior knowledge about the strength under assessment are seen to be essential aspects for the quantification of the CFs. The number of material tests is the key factor used to set the probabilistic framework of the present Section, while the influence of the existence of prior knowledge is addressed in Sect. 4. Within the scope of the study, it is also assumed that the CFs proposed by EC8-3 guarantee a certain level of reliability of the material strength value (after its adjustment by the $\mathrm{CF}$ ) that is associated to the minimum number of tests. Although the referred level of reliability is not easy to quantify, it is also addressed by the study by associating certain confidence levels to the quantification of the CFs. Furthermore, since the framework for the $\mathrm{CF}$ definition proposed by EC8-3 is material independent, the evaluation of the EC8-3 proposed CF values is 
illustrated herein for the case of RC structures. Nonetheless, part of the study is presented in a form independent of the material. Hence, it can be readily applied to any material and property.

As stated in the previous Section, when concrete compressive strength test results are referred hereon, they are assumed to be obtained from destructive tests (i.e. core compression tests). It is also assumed that such test results have been converted to the corresponding in-place concrete strength. As an extensive discussion on such procedures is beyond the scope of this work, the reader is referred, for example, to [15, 18-20] for further details. In terms of the number of tests, and based on Sect. 2.2, the critical situation occurs for a one-storey structure with beams and columns of different concrete grades as primary elements. To assess the strength of each concrete grade, the minimum number of tests can be interpreted as being one, two and three for each concrete grade for levels KL1, KL2 and KL3, respectively.

Two important assumptions are additionally considered. First, the statistical distribution of the concrete compressive strength is assumed to follow either a normal, lognormal or Weibull distribution [13, 2123]. Secondly, it is also assumed that strength variability, characterized herein by its coefficient of variation $(\mathrm{CoV})$, is within the range of 6-20\% [21, 23-26]. Although larger $\mathrm{CoV}$ values can be found in the literature [21, 24, 27], a maximum of $20 \%$ is already considered to be very high for normal strength concrete, either site-mixed or ready-mixed. Further discussion on this topic can be found elsewhere $[14,25]$.

The proposed framework for the probabilistic definition of the CFs is based on the concept of confidence interval (an interval of real numbers expected to contain the true value of a population parameter, with a specified confidence). Considering that $\theta$ is a population parameter to be estimated, $T$ as the statistic used as a point estimate for $\theta$ and denoting $\hat{\theta}$ as the observed value of the statistic, an interval estimate for $\theta$ has the form

$\hat{\theta}_{\mathrm{L}} \leq \theta \leq \hat{\theta}_{\mathrm{U}}$

where $\hat{\theta}_{\mathrm{L}}$ and $\hat{\theta}_{\mathrm{U}}$ are lower and upper bounds, respectively, of the confidence interval, computed from the sample data. Since different samples will produce different values of $\hat{\theta}_{\mathrm{L}}$ and $\hat{\theta}_{\mathrm{U}}$, these end-points are values of the random variables $\theta_{\mathrm{L}}$ and $\theta_{\mathrm{U}}$, respectively. By knowing the sampling distribution of $T$, it is possible to determine values of $\theta_{\mathrm{L}}$ and $\theta_{\mathrm{U}}, \hat{\theta}_{\mathrm{L}}$ and $\hat{\theta}_{\mathrm{U}}$ respectively, such that

$P\left(\hat{\theta}_{\mathrm{L}} \leq \theta \leq \hat{\theta}_{\mathrm{U}}\right)=1-\alpha$

where $0 \leq \alpha \leq 1$. This expression indicates there is a $(1-\alpha)$ probability of selecting a random sample producing an interval that contains $\theta$ and provides both lower and upper confidence bounds for $\theta$. One-sided confidence bounds are obtained by setting either $\hat{\theta}_{\mathrm{L}}=$ $-\infty$ or $\hat{\theta}_{\mathrm{U}}=\infty$.

\subsection{Definition of the CFs for the case of a normal distributed strength}

The proposed approach defines the CFs based on the confidence interval for the mean of the normal distribution with known variance [28]. As the present study focuses on assessing the adequacy of the EC8-3 proposed $\mathrm{CF}$ values, it is assumed that such values only account for the uncertainty in the estimation of the mean value and not for that of the variance. Hence the known variance hypothesis is favoured instead of the unknown variance one. Still, as shown in the following, the proposed approach does not require knowledge about the specific value of the variance, but instead demands for a measure of the relative variability which is defined by the $\mathrm{CoV}$. As stated in the previous Section, the characterization of the variability of the material property in terms of the $\mathrm{CoV}$ is defined using a range of realistic values given in the literature.

The consideration of the unknown variance hypothesis presents some difficulties for the present study. For example, it does not allow the definition of the $\mathrm{CF}$ value for samples of size equal to one. Additionally, for samples of smaller size, the sample variance (or sample $\mathrm{CoV}$ ) is known to be a poor estimator of the true variance and has a high sensitivity to potentially anomalous observations [29]. Therefore, the definition of an adequate range for the expected values of the sample $\mathrm{CoV}$ is not as straightforward as for the known variance case. To clarify these issues and to observe the practical implications of considering this hypothesis in the context of the definition of CF values, the unknown variance case is also addressed herein. 


\subsubsection{The case of a normal distributed strength with known variance}

Considering that $X_{1}, X_{2}, \ldots X_{n}$ is a random sample drawn from a normal distribution with unknown mean $\mu$ and known standard deviation $\sigma$, the sample mean $\bar{X}$ is known to be normally distributed with mean $\mu$ and standard deviation $\sigma / \sqrt{n}$ [28]. By standardizing $\bar{X}$ one obtains variable $Z$ :

$Z=\frac{\bar{X}-\mu}{\sigma / \sqrt{n}}$

which follows a standard normal distribution [28] and leads to:

$P\left(-z_{1-\alpha / 2} \leq \frac{\bar{X}-\mu}{\sigma / \sqrt{n}} \leq z_{1-\alpha / 2}\right)=1-\alpha$

where $z_{1-\alpha / 2}$ is the $(1-\alpha / 2)$ percentage point of the standard normal distribution. The one-sided lower bound expression equivalent to Eq. (5) is given by

$P\left(\frac{\bar{X}-\mu}{\sigma / \sqrt{n}} \leq z_{1-\alpha}\right)=1-\alpha$

where $z_{1-\alpha}$ is the $(1-\alpha)$ percentage point of the standard normal distribution.

Based on the previously established critical safety situation, the definition of an adequate $\mathrm{CF}$ value must verify the following condition:

$\bar{X} / \mathrm{CF} \leq \mu \Leftrightarrow \mathrm{CF} \geq \bar{X} / \mu$

The minimum CF value that verifies Eq. (7) is:

$\mathrm{CF}=\bar{X} / \mu$

Combining Eq. (6) with Eq. (8) yields

$P\left(\mathrm{CF} \leq 1+z_{1-\alpha} \cdot \mathrm{CoV} / \sqrt{n}\right)=1-\alpha$

where the $\mathrm{CoV}$ is $\sigma / \mu$ and states that, for a known (expected) value of the $\mathrm{CoV}$, there is a $(1-\alpha)$ probability that $\mathrm{CF} \leq 1+z_{1-\alpha} \cdot \mathrm{CoV} / \sqrt{n}$ if $\mu=$ $\bar{X} / \mathrm{CF}$. Therefore, the $(1-\alpha) \cdot 100 \%$ upper confidence bound on the value of $\mathrm{CF}$ is:

$\mathrm{CF} \leq 1+z_{1-\alpha} \cdot \mathrm{CoV} / \sqrt{n}$

In order to set the CF values, one is interested in the limiting values given by Eq. (10), hereafter termed $\mathrm{CF}_{\text {LIM }}$, for increasing values of the number of tests $n$, for a prescribed $(1-\alpha)$ confidence level and a given
$\mathrm{CoV}$. In the situation of assessing the adequacy of the CFs recommended by EC8-3, the definition of a single $\mathrm{CF}$ value for each KL must account for the most unfavourable conditions, namely in terms of number of tests and CoV. Although critical situations can be identified for these two parameters based on the previously exposed arguments, there is little guidance for which $(1-\alpha)$ confidence level should be chosen. Even though there is no apparent justification, a minimum confidence level of $75 \%$ is commonly considered in the assessment of existing structures context, e.g. [16, 30, 31]. Other authors propose to select the confidence level according to the importance of the structure defining levels of 75, 85-90 and $95 \%$ for ordinary, important and very important structures, respectively, [32, 33]. For the study presented herein, the confidence levels must be defined as a function of the KLs set by EC8-3, thus reflecting the minimum number of material tests required. Assuming a minimum confidence level of $75 \%$ and considering that a confidence level of $95 \%$ is sufficiently large for practical purposes, confidence levels of 95, 85 and $75 \%$ are proposed for KL1, KL2 and KL3, respectively. These values are set considering that when KL increases, the uncertainty about the materials decreases, thus the amplitude of the confidence interval, which is defined by the confidence level and reflects the uncertainty, can be smaller.

In order to observe the evolution of the $\mathrm{CF}_{\mathrm{LIM}}$ values for levels KL1, KL2 and KL3, Fig. 1a, b, and c present the upper limits of the confidence interval of the $\mathrm{CF}$ defined by Eq. (10), given the condition $\mu=\bar{X} / \mathrm{CF}$. These are defined for increasing values of $n$ (from 1 to 30 ), for the previously defined range of the $\mathrm{CoV}$ (6-20\% in $2 \%$ steps) and for the corresponding confidence levels $(75,85$ and $95 \%)$. Each graph presents also the computed $\mathrm{CF}_{\text {LIM }}$ corresponding to the larger $\mathrm{CoV}$ and to the minimum number of tests of the corresponding KL (underlined value). As expected, the analysis of the computed CFs indicates that, irrespective of the selected confidence level, $\mathrm{CF}_{\text {LIM }}$ tends asymptotically to 1.0 as $n$ tends to infinity. Moreover, the analysis of the underlined values shows that, for the previously set of hypotheses, the CF values proposed by EC8-3 for KL1 and KL2 seem adequate, while for KL3 there is a significant difference between the proposed value and $\mathrm{CF}_{\mathrm{LIM}}$. Although approaching 1.0 for a very large $n$, such 

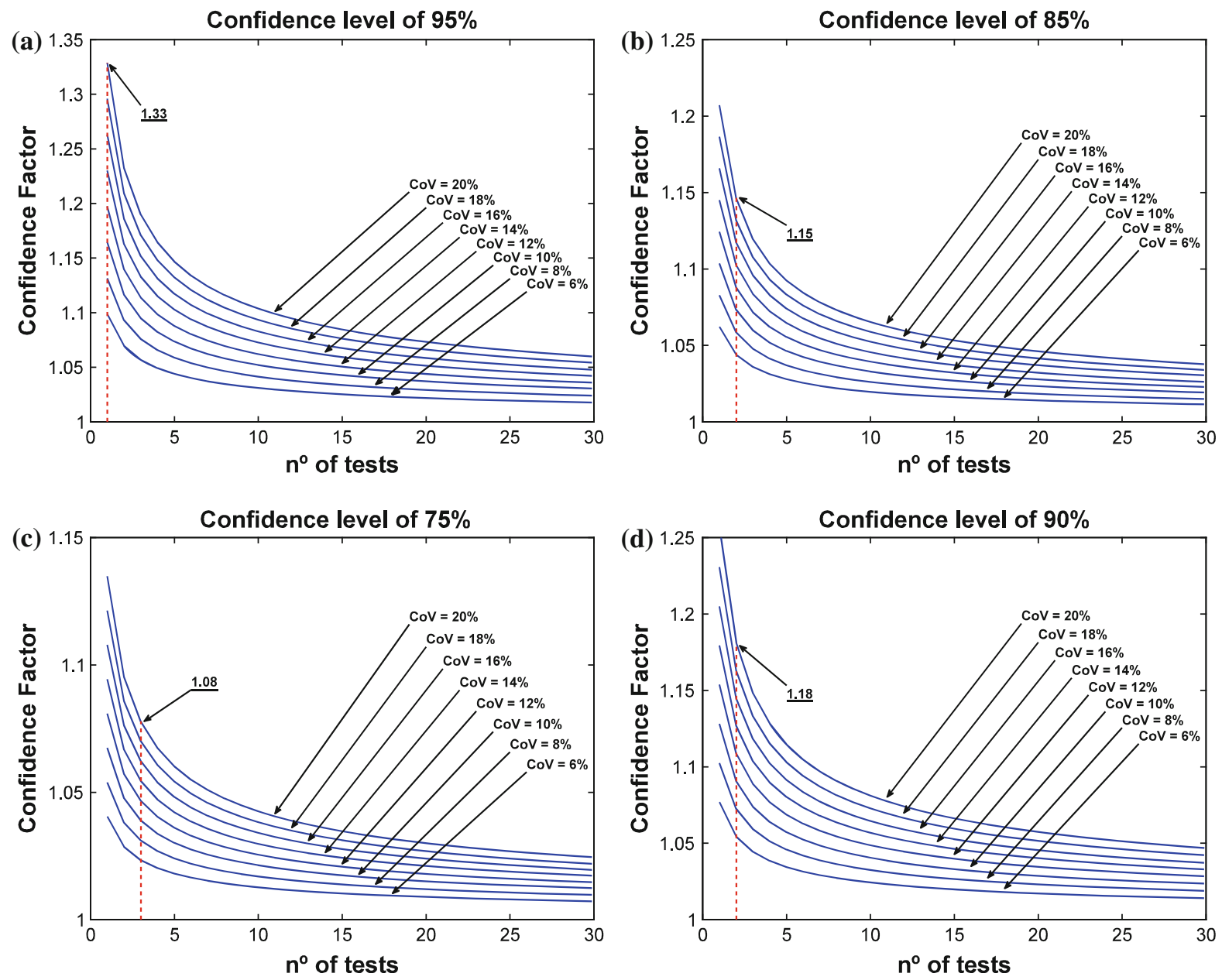

Fig. 1 Values of $\mathrm{CF}_{\mathrm{LIM}}$ considering a normal distributed strength, for increasing values of $n$, for the selected range of CoVs and for $(1-\alpha)$ confidence levels of $95 \%(\mathbf{a}), 85 \%(\mathbf{b}), 75 \%(\mathbf{c})$ and $90 \%(\mathbf{d})$

value of $\mathrm{CF}_{\text {LIM }}$ can only be attained for all values of $n$ by setting the confidence level to $50 \%$, for which the term $z_{1-\alpha}$ of Eq. (10) is zero. With respect to KL2, given the difference between $\mathrm{CF}_{\mathrm{LIM}}$ (i.e. 1.15) and the EC8-3 proposed value (i.e. 1.2), $\mathrm{CF}_{\text {LIM }}$ value was also computed for a confidence level of $90 \%$. Figure 1d shows that, for the aforementioned conditions and for a confidence level of $90 \%$, the $\mathrm{CF}_{\text {LIM }}$ value obtained is closer to the EC8-3 proposal. On the basis of this result, subsequent analyses will consider a confidence level of $90 \%$ for KL2.

\subsubsection{The case of a normal distributed strength with unknown variance}

For comparison purposes, the case of the normal distributed strength with unknown variance is considered herein and the corresponding expression for the upper confidence bound on the value of $\mathrm{CF}$ is also established. For a normal distribution with unknown mean and unknown standard deviation, the random variable $T$ defined by

$T=\frac{\bar{X}-\mu}{s / \sqrt{n}}$

where $s$ is the sample standard deviation, is known to follow a $t$ distribution with $n-1$ degrees of freedom [28]. The one-sided lower bound expression equivalent to Eq. (5) is now given by

$P\left(\frac{\bar{X}-\mu}{s / \sqrt{n}} \leq t_{1-\alpha, n-1}\right)=1-\alpha$

where $t_{1-\alpha, n-1}$ is the $(1-\alpha)$ percentage point of the $t$ distribution with $n-1$ degrees of freedom. Following 

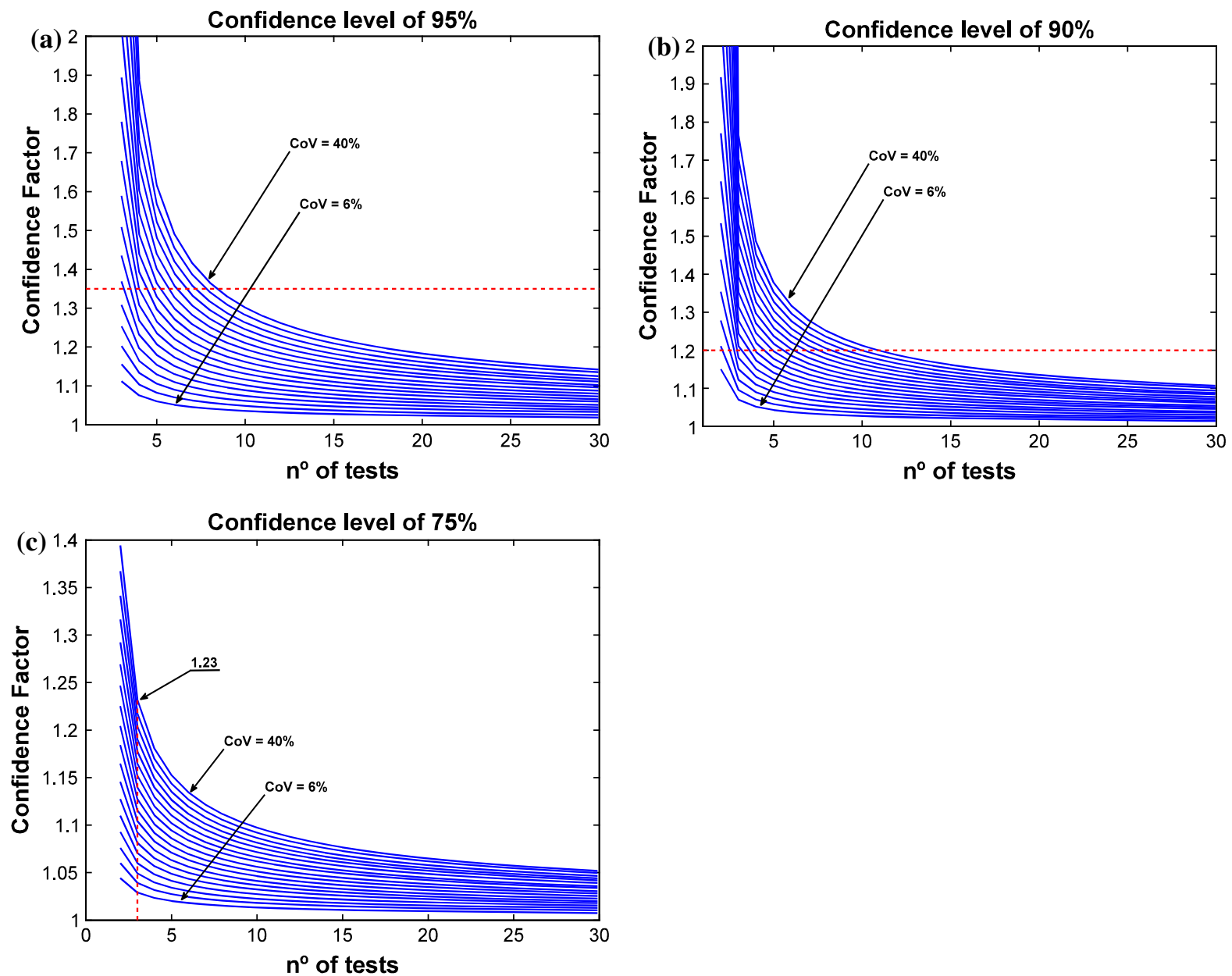

Fig. 2 Values of $\mathrm{CF}_{\mathrm{LIM}}$ considering a normal distributed strength with unknown variance, for increasing values of $n$, for the selected range of $\overline{\mathrm{CoV}} \mathrm{s}$ and for $(1-\alpha)$ confidence levels of $95 \%$ (a), $90 \%$ (b) and $75 \%$ (c)

the same reasoning as for the previous case and considering that $\overline{\mathrm{CoV}}$ is the sample $\mathrm{CoV}$ defined by $s / \bar{X}$, the $(1-\alpha) \cdot 100 \%$ upper confidence bound for the value of $\mathrm{CF}$ is now obtained by:

$\mathrm{CF} \leq\left(1-t_{1-\alpha, n-1} \cdot \overline{\mathrm{CoV}} / \sqrt{n}\right)^{-1}$

In order to observe the evolution of the $\mathrm{CF}_{\mathrm{LIM}}$ values using Eq. (13), adequate values of the $\overline{\mathrm{CoV}}$ must first be defined. Since for samples of small size, as those considered herein, the values of the $\mathrm{CoV}$ and of the $\overline{\mathrm{CoV}}$ can be significantly different, the range of the $\overline{\mathrm{CoV}}$ is considered to be wider than that of the $\mathrm{CoV}$. To determine a possible upper limit for the referred $\overline{\mathrm{CoV}}$ range, a simulation study was performed. The simulation started with the selection of a concrete class characterized by having a compressive strength following a normal distribution with a $\mathrm{CoV}$ of $20 \%$ and a $\mu$ selected from the range of $12-50 \mathrm{MPa}$, in $1 \mathrm{MPa}$ steps. Afterwards, 50,000 samples of size two were randomly drawn from the normal distribution and the $\overline{\mathrm{CoV}}$ was determined for each one. After computing the $\overline{\mathrm{CoV}}$ for all samples, considering all possible values of $\mu$ from the previously set range, the 95th quantile of the corresponding empirical cumulative distribution function (CDF), which is around $40 \%$, was selected as the upper limit for the $\overline{\mathrm{CoV}}$. Therefore, the selected range of the $\overline{\mathrm{CoV}}$ was $6-40 \%$, in $2 \%$ steps. Considering this range, Fig. 2a, b, and c present the $\mathrm{CF}_{\text {LIM }}$ values obtained from Eq. (13), given the condition $\mu=\bar{X} / \mathrm{CF}$ and for levels KL1, 
KL2 and KL3. As previously referred, results for $n=$ 1 are not available and, for the case of KL1, Fig. 2a, results for $n=2$ are also not presented because the term $t_{1-\alpha, n-1} \cdot \overline{\mathrm{CoV}} / \sqrt{n}$ is larger than 1.0 for $\overline{\mathrm{CoV}}$ values above $22 \%$. This situation reflects the possibility of a negative value of $\mu$, which is not admissible. Observation of Fig. 2 confirms the expected increase of the $\mathrm{CF}_{\text {LIM }}$ values for small values of $n$. According to Fig. 2a, b, for KL1 and KL2, and for the upper value of the $\overline{\mathrm{CoV}}$ range, the minimum number of tests that validate the $\mathrm{CF}$ values proposed by EC8-3 now need to be nine and eleven, respectively. For KL3, Fig. 2c), it can be seen that the $\mathrm{CF}_{\mathrm{LIM}}$ corresponding to the larger $\overline{\mathrm{CoV}}$ and to the minimum number of tests $(n=3)$ is now 1.23, when for the known variance case such value is 1.08. As can be seen from the results, the unknown variance case does not lead to values of the minimum number of tests (or of the CFs) that are in agreement with the EC8-3 proposals. Hence, it is believed that the known variance hypothesis may be underlining the $\mathrm{CF}$ definition in the context of the EC8-3 safety assessment procedures.

\subsection{Definition of the CFs for the case of a lognormal distributed strength}

As for the normal distribution case, the definition of the CFs is performed for the known and the unknown variance assumptions. Although the former hypothesis is considered to be more relevant for the EC8-3 context, the latter is considered for comparison purposes, in order to observe the variation of the $\mathrm{CF}$ values due to the uncertainty of the variance.

\subsubsection{The case of a lognormal distributed strength with known variance}

For this case, the CFs can be characterized following the approach of the normal distribution with known variance. Considering that $Y_{1}, Y_{2}, \ldots Y_{n}$ is a random sample from a lognormal distribution with unknown mean $\theta$ and known standard deviation $\delta$, the variable $X=\ln (Y)$ follows a normal distribution with mean $\mu$ and standard deviation $\sigma$. As for the case of the normal distribution, the proposed approach does not require knowledge about the specific value of $\delta$, but instead demands for a measure of the relative variability which is defined by the CoV. Upon this, it follows that
Eq. (6) is applicable and can be rearranged to give the $(1-\alpha)$ probability that

$\bar{X}-z_{1-\alpha} \cdot \sigma / \sqrt{n} \leq \mu$

which, by adding $\sigma^{2} / 2$ on both sides and taking exponentials of both sides, leads to

$e^{\left(\bar{X}+\frac{\sigma^{2}}{2}\right)} \cdot e^{-z_{1-\alpha} \cdot \frac{\sigma}{\sqrt{n}}} \leq e^{\left(\mu+\frac{\sigma^{2}}{2}\right)}$

Knowing that $\theta$ is given by

$\theta=e^{\left(\mu+\frac{\sigma^{2}}{2}\right)}$

and considering $\bar{Y}$ to be its sample estimate, yields

$\bar{Y} \cdot e^{-z_{1-\alpha \cdot \frac{\sigma}{\sqrt{n}}}} \leq \theta$

Considering a reasoning similar to that of Eq. (7):

$\bar{Y} / \mathrm{CF} \leq \theta \Leftrightarrow \mathrm{CF} \geq \bar{Y} / \theta$

Similar to Eq. (8), the minimum CF value that verifies Eq. (18) is:

$\mathrm{CF}=\bar{Y} / \theta$

Combining Eq. (17) with Eq. (19) and rearranging leads to

$\mathrm{CF} \leq e^{z_{1-\alpha} \cdot \sqrt{\frac{\ln \left(\mathrm{CoV}^{2}+1\right)}{n}}}$

where $\sqrt{\ln \left(\mathrm{CoV}^{2}+1\right)}$ is $\sigma$. As in Eq. (10), Eq. (20) gives the upper confidence bound $\mathrm{CF}_{\mathrm{LIM}}$ for which there is a $(1-\alpha)$ probability that $\mathrm{CF} \leq e^{z_{1-\alpha} \cdot \sqrt{\frac{\ln \left(\mathrm{CoV}^{2}+1\right)}{n}}}$ if $\theta=\bar{Y} / \mathrm{CF}$.

As for the case of the normal distribution, evolutions of $\mathrm{CF}_{\text {LIM }}$ can be obtained simulating Eq. (20) for increasing values of $n$, for the previously defined range of the $\mathrm{CoV}$ and for the confidence levels of each KL. For the sake of brevity, graphical representations of the referred evolutions are not presented herein. Nonetheless, it should be noted that Eq. (20) gives larger $\mathrm{CF}_{\mathrm{LIM}}$ values than Eq. (10). For the larger $\mathrm{CoV}$ $(20 \%)$ and for the minimum number of tests of each $\mathrm{KL}$, the $\mathrm{CF}_{\mathrm{LIM}}$ values are $1.39,1.20$ and 1.08 for KL1, KL2 and KL3, respectively. When comparing these results with the $\mathrm{CF}$ values proposed by $\mathrm{EC} 8-3$, it is seen that only the value of KL2 agrees with the EC8-3 proposal. In order for the $\mathrm{CF}_{\mathrm{LIM}}$ of KL1 to meet the EC8-3 proposed value (i.e. 1.35), there is the need to either reduce the prescribed confidence level or to 
reduce the maximum admissible $\mathrm{CoV}$. Therefore, one of the following two situations can be observed:

When fixing the $\mathrm{CoV}$ to $20 \%$ and when $n$ is one, the $(1-\alpha)$ confidence level that yields a $\mathrm{CF}_{\text {LIM }}$ of 1.35 is $93.5 \%$;

When fixing the $(1-\alpha)$ confidence level to $95 \%$ and when $n$ is one, the $\mathrm{CoV}$ that yields a $\mathrm{CF}_{\text {LIM }}$ of 1.35 is $18.5 \%$.

Considering that the observed reduction can be seen to be relatively small, one is inclined to validate the adequacy of the EC8-3 proposal for KL1. Moreover, considering that the number of tests is fixed to one in both cases, a number that will most surely be exceeded in real situations, it is worth doing the analysis of the $\mathrm{CF}_{\text {LIM }}$ value when the number of tests is set to two. In this case, the $\mathrm{CF}_{\mathrm{LIM}}$ value for a $\mathrm{CoV}$ of $20 \%$ and a confidence level of $95 \%$ is now 1.26 , well below the EC8-3 proposal for this KL.

\subsubsection{The case of a lognormal distributed strength with unknown variance}

Statistical methods for inference involving the lognormal mean, namely addressing the estimation of confidence intervals when both mean and variance are unknown, have received widespread attention in the literature over the years. Available methods for the development of such confidence intervals range from analytical, or semi-analytical, procedures to computationally intensive simulation approaches [34-40]. From observation of the available procedures, their complexity can be seen to be larger than that of the previously presented approaches. Although research on this topic appears to be far from over, the analytical method proposed by [40] is seen to yield adequate results for small samples and presents a suitable form for the purpose of the present study.

According to [40], the one-sided lower bound LB of the $(1-\alpha)$ confidence interval for the lognormal mean $\theta$ is defined by

$\mathrm{LB}=\bar{M} \cdot e^{-\left(\frac{z_{1-\alpha^{s^{2}}}}{n}+\left(\frac{s^{2}}{2}-\frac{(n-1) s^{2}}{2 \chi_{1-\alpha, n-1}^{2}}\right)^{2}\right)}$

where $s^{2}$ is the sample variance of the log-transformed data, $\chi_{1-\alpha, n-1}^{2}$ is the $(1-\alpha)$ percentage point of the $\chi^{2}$ distribution with $n-1$ degrees of freedom and $\bar{M}$ is the sample mean given by
$\bar{M}=e^{\left(\bar{X}+\frac{s^{2}}{2}\right)}$

Following the same reasoning as for the previous cases and considering that $\overline{\mathrm{CoV}}$ is the sample $\mathrm{CoV}$ now defined by $\sqrt{e^{s^{2}}-1}$, the $(1-\alpha) \cdot 100 \%$ upper confidence bound on the value of CF is now obtained by:

$\mathrm{CF} \leq e^{\left[\frac{z_{1-\alpha}^{2} \ln \left(\overline{\mathrm{cov}}^{2}+1\right)}{n}+\left(\frac{\ln \left(\overline{\mathrm{Cov}}^{2}+1\right)}{2}\left(1-\frac{(n-1)}{\chi_{1-\alpha, n-1}^{2}}\right)\right)^{2}\right]^{0.5}}$

As for the normal distribution with unknown variance case, to observe the evolution of the $\mathrm{CF}_{\mathrm{LIM}}$ values using Eq. (23), adequate values of the $\overline{\mathrm{CoV}}$ must also be defined. A simulation study similar to that of the normal distribution case was performed and the resulting 95th quantile of the $\overline{\mathrm{CoV}} \mathrm{CDF}$ was also seen to be near $40 \%$. Hence, the selected range for the $\overline{\mathrm{CoV}}$ was also considered to be $6-40 \%$, in $2 \%$ steps, for this case.

Considering this range, Fig. 3a, b, and c present the $\mathrm{CF}_{\text {LIM }}$ values obtained from Eq. (23), given the condition $\theta=\bar{M} / \mathrm{CF}$ and for levels KL1, KL2 and KL3. As for the normal distribution case, results for $n=1$ are not available. Observation of Fig. 3 also confirms the expected increase of the $\mathrm{CF}_{\mathrm{LIM}}$ values for small values of $n$ when compared to those of the known variance case. According to Fig. 3a, b, for KL1 and KL2, and for the upper value of the $\overline{\mathrm{CoV}}$ range, the minimum number of tests that validate the $\mathrm{CF}$ values proposed by EC8-3 now need to be five and eight, respectively. For KL3, Fig. 3c, it can be seen that the $\mathrm{CF}_{\text {LIM }}$ corresponding to the larger $\overline{\mathrm{CoV}}$ and to the minimum number of tests $(n=3)$ is now 1.16 , when for the known variance case such value is 1.08 . By comparing these results with those of Sect. 3.4, it can be seen that considering the normal distribution with unknown variance yields more conservative results. On the other hand, when the variance uncertainty is not taken into account, it is the lognormal distribution that yields more conservative $\mathrm{CF}$ values. This change of status between the normal and the lognormal distributions results from the fact that Eq. (21) includes the contribution of the sampling distribution of $s^{2}$ which is a right-skewed Chi-square distribution. Hence, the lognormal distribution hypothesis leads to unsymmetrical right-skewed two-sided confidence intervals while symmetric ones are obtained with the normal distribution assumption [40]. 

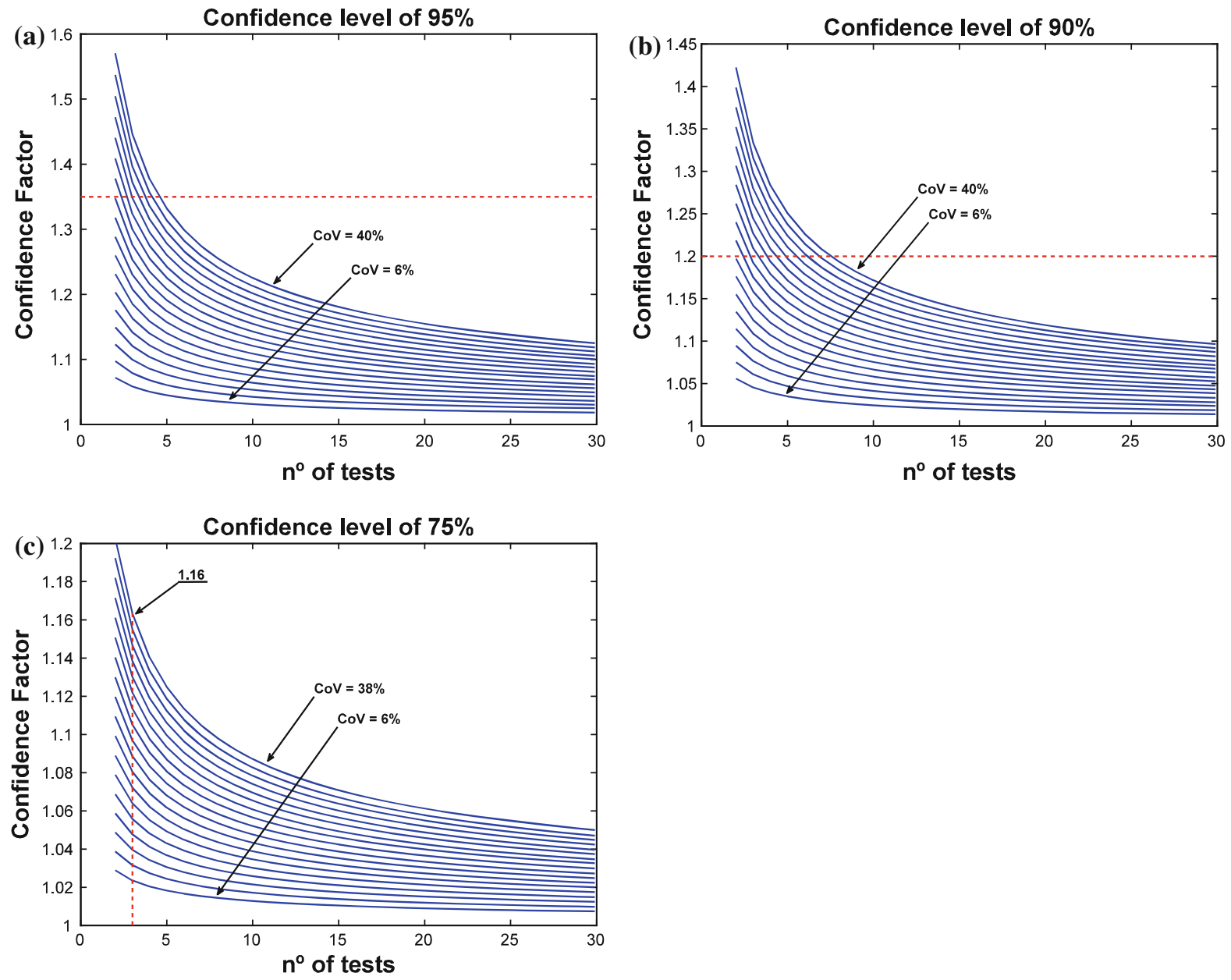

Fig. 3 Values of $\mathrm{CF}_{\mathrm{LIM}}$ considering a lognormal distributed strength with unknown variance, for increasing values of $n$, for the selected range of $\overline{\mathrm{CoVs}}$ and for $(1-\alpha)$ confidence levels of $95 \%(\mathbf{a}), 90 \%$ (b) and $75 \%$ (c)

\subsection{Definition of the CFs for the case of a Weibull distributed strength}

The two-parameter Weibull distribution [41] defined by the CDF of the form

$F(x)=1-e^{-(x / \beta)^{\gamma}}$

where $\gamma$ and $\beta$ are, respectively, the shape and the scale parameters, was chosen to characterize the CFs in the case of a Weibull distributed strength [23]. Unlike for the case of the normal and the lognormal distributions, mathematically tractable confidence intervals are not available for the mean $\mu$ of the Weibull distribution. For the case of large samples, it is possible to assume that the distribution of the mean estimate $\bar{X}$ is asymptotically normal [28]. However, this assumption is not applicable for the present case. Several alternatives providing confidence intervals for $\mu$ have been proposed considering the Weibull-to-exponential transform or considering Type II censored data [4244]. Still, none of these approaches were found to be attractive due to the difficulty of their application to the present case. Given the simplicity of using a simulation approach, this method was selected to assess the $\mathrm{CF}_{\mathrm{LIM}}$ values for the Weibull distribution case.

The simulation method started with the selection of a concrete class characterized by a compressive strength with chosen $\mu$ and $\mathrm{CoV}$, the former being selected from the range of 12 to $50 \mathrm{MPa}$, in $1 \mathrm{MPa}$ steps, and the latter being selected from the previously set range, considering $2 \%$ steps. Knowing $\mu$ and $\mathrm{CoV}$, 

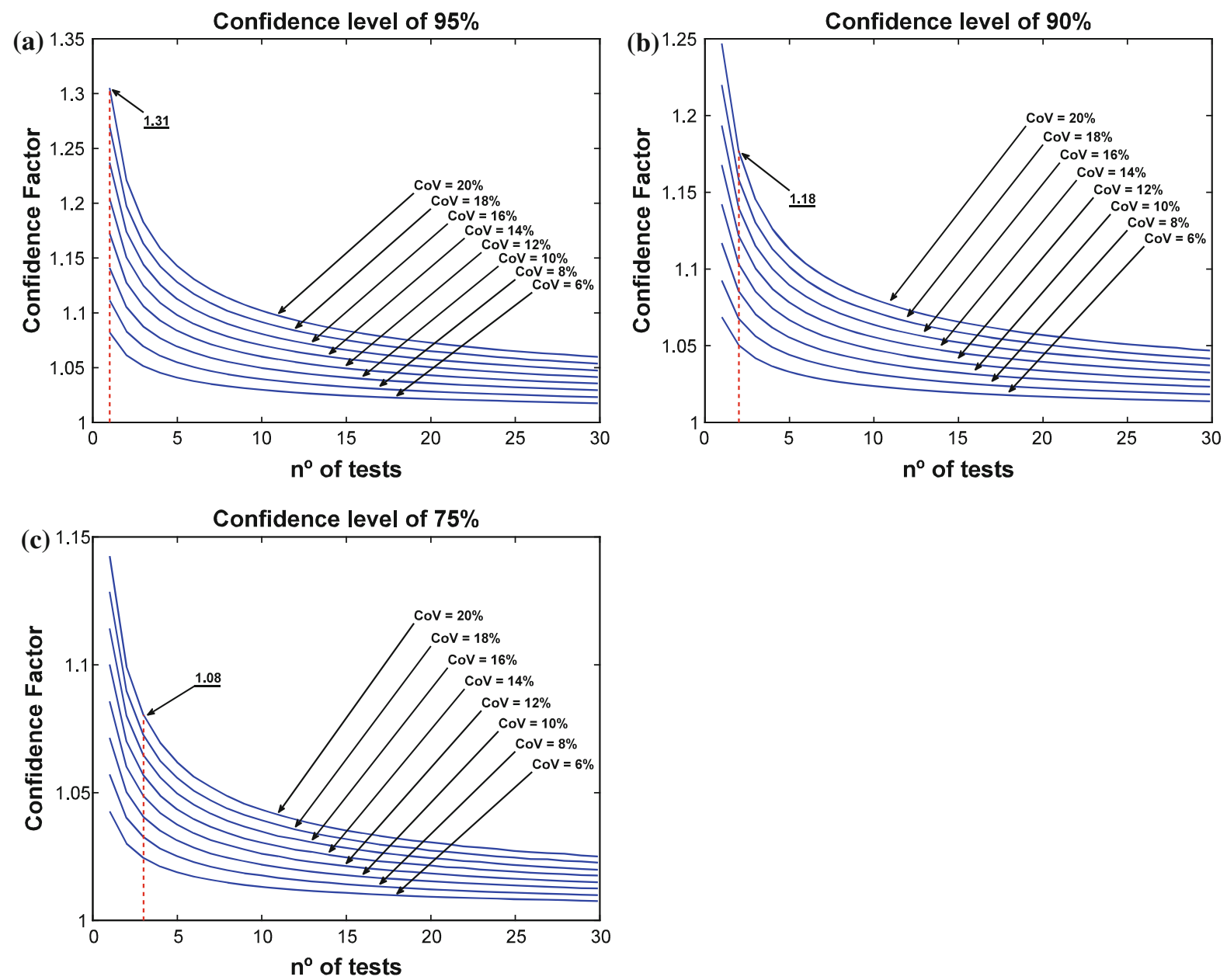

Fig. 4 Values of $\mathrm{CF}_{\mathrm{LIM}}$ considering a Weibull distributed strength, for increasing values of $n$, for the selected range of CoVs and for $(1-\alpha)$ confidence levels of a $95 \%, \mathbf{b} 90 \%$ and $\mathbf{c} 75 \%$

the Weibull parameters $\gamma$ and $\beta$ are determined based on the following relations:

$\mu=\beta \cdot \Gamma(1+1 / \gamma)$

$\operatorname{CoV}=\frac{\left[\Gamma(1+2 / \gamma)-\Gamma^{2}(1+1 / \gamma)\right]^{1 / 2}}{\Gamma(1+1 / \gamma)}$

where $\Gamma($.$) is the Gamma function. Based on Eq. (26),$ parameter $\gamma$ can be determined for the known $\mathrm{CoV}$ using a standard Newton-Raphson method after which parameter $\beta$ can be obtained using Eq. (25). Afterwards, 50,000 samples of a chosen size $n$ were randomly drawn from the Weibull distribution. Next, the mean value of each sample $i$ was computed and divided by $\mu$ to yield the $\mathrm{CF}_{i}$, i.e. the $\mathrm{CF}$ value of sample $i$. After computing $\mathrm{CF}_{i}$ values for all samples, considering all possible values of $\mu$ from the previously set range and for a given $\mathrm{CoV}$, an empirical $\mathrm{CDF}$ was defined, for which $\mathrm{CF}_{\mathrm{LIM}}$ corresponds to the $(1-\alpha)$ percentile. The simulation process was then repeated for different values of size $n$, from 1 to 30 , and for the several $\mathrm{CoV}$ values from their previously set range. Since the $\mathrm{CoV}$ range was considered, instead of the $\overline{\mathrm{CoV}}$ one, the simulation is assumed to represent a known variance situation. The unknown variance hypothesis was not considered for the Weibull distribution case.

In order to observe the evolution of the $\mathrm{CF}_{\mathrm{LIM}}$ values for levels KL1, KL2 and KL3, Fig. 4a, b, and c present the results obtained from the referred simulation study for increasing values of $n$ (from 1 to 30), for the previously defined range of the $\mathrm{CoV}(6-20 \%$ in $2 \%$ steps) and for the corresponding confidence levels $(75,90$ and $95 \%)$. As for the case of the normal 
Table 3 Analysis of the CF values for different building sizes, considering different distributions, KLs and variance knowledge hypotheses ( $K V$ known variance, $U V$ unknown variance)

\begin{tabular}{|c|c|c|c|c|c|c|c|}
\hline \multirow[t]{2}{*}{ Knowledge level } & \multirow[t]{2}{*}{ Distribution } & \multicolumn{2}{|c|}{1 storey } & \multicolumn{2}{|c|}{3 storeys } & \multicolumn{2}{|c|}{5 storeys } \\
\hline & & KV & UV & KV & UV & KV & UV \\
\hline \multirow[t]{2}{*}{ KL1 } & Normal & 1.35 & - & 1.13 & 1.49 & 1.10 & 1.30 \\
\hline & Lognormal & 1.35 & - & 1.14 & 1.30 & 1.11 & 1.23 \\
\hline \multirow[t]{2}{*}{ KL2 } & Normal & 1.20 & - & 1.07 & 1.19 & 1.06 & 1.13 \\
\hline & Lognormal & 1.20 & - & 1.08 & 1.16 & 1.06 & 1.12 \\
\hline \multirow[t]{2}{*}{ KL3 } & Normal & 1.08 & - & 1.03 & 1.07 & 1.02 & 1.05 \\
\hline & Lognormal & 1.08 & - & 1.03 & 1.06 & 1.02 & 1.05 \\
\hline
\end{tabular}

distributed strength with known variance, the computed $\mathrm{CF}_{\mathrm{LIM}}$ corresponding to the larger $\mathrm{CoV}$ and to the minimum number of tests of the corresponding $\mathrm{KL}$ is also represented in each graph (underlined value). The analysis of the underlined values shows that, for the previous set of hypotheses, the $\mathrm{CF}$ values proposed by EC8-3 for KL1 and KL2 appear to be adequate while the proposed value for KL3 is significantly different from the obtained $\mathrm{CF}_{\mathrm{LIM}}$, as reported for the case of normal and lognormal distributed strengths.

\subsection{Discussion of the results}

Based on the results obtained for the different distributions and assumptions considered in the presented study, the EC8-3 proposed CFs for KL1 and KL2 are believed to be adequate for the purpose of establishing a conservative value of the material strength, assuming that the selected confidence levels are satisfactory. Moreover, the comparison of the results obtained with the $\mathrm{CF}$ values proposed by EC8-3 are seen to be more consistent with the known variance assumption. With respect to KL3, the EC83 proposed CF is not met by any of the cases studied. Nonetheless, the known variance assumption is also selected since it leads to lower values of the CF. For this case, a CF value of 1.08 is seen to be more adequate to the assumptions made in the study. Still, it is recalled that, for KL3, a CF value of 1.0 can only be obtained if the confidence level is assumed to be $50 \%$.

The aforementioned conclusions are based on a limit $\mathrm{CoV}$ of $20 \%$ and for a number of tests corresponding to the minimum values established according to the conditions referred in Sect. 2.2. Nonetheless, it is useful to analyse such results in terms of the necessary $\mathrm{CF}$ associated to building structures of more common sizes. For example, let us consider three- and five-storey framed structures having a single concrete grade and only with beams and columns as primary elements. According to Table 1, the minimum number of tests for KL1, KL2 and KL3 are now 6, 12 and 18, respectively, for the three-storey building, and 10, 20 and 30, respectively, for the five-storey building. By determining the $\mathrm{CF}$ values for such number of tests, for the normal and the lognormal distributions (the Weibull distribution results are similar to those of the normal distribution), considering the known variance hypothesis and the limit $\mathrm{CoV}$ of $20 \%$, the results presented in Table 3 are obtained (results for a one-storey structure are also presented for comparison). As can be seen, in comparison with the CF values of the one-storey building, CFs could be significantly reduced for the three-storey structure. On the other hand, going from the three- to the five-storey buildings implies a negligible reduction of the CF values. Hence, for buildings higher than three-storeys, significantly reduced $\mathrm{CF}$ values could be considered instead. A similar analysis considering the unknown variance hypothesis and for the $\overline{\mathrm{CoV}}$ of $40 \%$ leads to the second set of CF values of Table 3 . In this case, the EC8-3 proposed CF for KL2 can be seen to also include the unknown variance hypothesis for buildings higher than three-storeys. Moreover, it can also be seen that, for KL3, the proposed value of 1.08 is also able to include such hypothesis under the same conditions. On the other hand, for the case of KL1, this situation can only be attained if the maximum $\overline{\mathrm{CoV}}$ is reduced to $32 \%$. 


\section{Quantification of the CFs when prior knowledge exists}

The situation of existing prior knowledge about the materials covers cases where design data or previous testing data is available. It is also referred that, for practical purposes, this latter case is the same as that of combining testing data coming from different types of tests (i.e. the case previously defined as Scenario 2). When there is prior knowledge about the materials based on available design data or previous testing data, EC8-3 accounts for this information to produce a more accurate estimate of the material strength and allows the required number of tests of a given $\mathrm{KL}$ to be reduced. By considering only the situation of material characterization and by observing the information provided in Table 1 with respect to the materials, the existence of original design specifications can be seen to imply that KL2 can be selected and there is only the need to carry out a minimum number of tests which corresponds to that of KL1. This value of the minimum number of tests implies a $50 \%$ reduction of the required number of tests, when compared to the situation of no prior knowledge (see Table 2). Furthermore, the existence of original test reports can be seen to imply that KL3 can be selected and there is only the need to carry out a minimum number of tests which corresponds also to that of KL1. In this case, a $67 \%$ reduction in the number of tests can be achieved when compared to the situation where there is no prior knowledge (see Table 2).

For a more clarified interpretation of the effects of existing prior knowledge in the context of the EC8-3 and its influence on the value of the CFs, an example analysis is presented in the following, considering the case of a normal distributed strength, the levels KL2 and KL3, and using a Bayesian formulation [45, 46]. Moreover, an additional example is presented for the case where testing data coming from different types of tests is combined, considering also a normal distributed strength and the previously referred Bayesian formulation. The reader is also referred to $[45,46]$ for information regarding the consideration of alternative distributions. The examples presented in the following represent a possible interpretation of the code procedures but are not an extensive study of the case of existing prior knowledge. Nonetheless, a qualitative evolution trend of the CF values can be observed from the following results.

\subsection{Considering prior knowledge and KL2}

When KL2 is chosen in the context of a situation where prior knowledge exists, it is assumed that original design specifications can lead to the definition of a prior estimate of the mean of the material strength, $X_{0}$. In this case, the updated estimate of the mean value of the material strength $X_{\mathrm{U}}$ can be obtained using a Bayesian approach given by $[45,46]$ :

$X_{\mathrm{U}}=\frac{n_{0} \cdot X_{0}+n_{1} \cdot X_{1}}{n_{0}+n_{1}}$

where $n_{0}$ is the number of tests considered for the estimate $X_{0}, X_{1}$ is the material strength estimate obtained from new data and $n_{1}$ is the number of tests that led to $X_{1}$. Since $X_{0}$ is based on design specifications and not on actual tests, $n_{0}$ must represent an equivalent number of tests. Although the adoption of other values could be foreseen, $n_{0}$ can be set to be the same as $n_{1}$ in order to guarantee the same level of reliability as when KL2 is considered with no prior knowledge (i.e. $n_{0}+n_{1}$ reflects the number of tests that would be considered for that situation with no prior knowledge). Upon this consideration, the value of $X_{\mathrm{U}}$ can therefore be seen to correspond to the average of $X_{0}$ and $X_{1}$. Given the weight of the prior estimate $X_{0}$ on the value $X_{\mathrm{U}}$ (i.e. $50 \%$, given that $n_{0}$ is equal to $n_{1}$ ), it should be emphasized that, in case the information between prior and new data $\left(X_{1}\right)$ is contradictory, the analyst has to decide whether the prior data should or should not be taken into account.

In order to observe the effects of existing prior knowledge on the quantification of the CF for KL2, $\mathrm{CF}_{\text {LIM }}$ values were assessed using a simulation similar to the one considered in Sect. 3.4, considering the same ranges for the concrete compressive strength and for the $\mathrm{CoV}$, but for the case of a Normal distribution. To account for the existence of prior knowledge, an extra step was included in the method after the computation of the mean value of each sample $i$. This step consists of the calculation of the updated mean $X_{\mathrm{U}}$ according to Eq. (27), which is then divided by $\mu$ to yield the $\mathrm{CF}_{i}$ as referred in Sect. 3.4. The outcome of the process is the empirical $\mathrm{CDF}$ of the $\mathrm{CF}_{i} s$, for which $\mathrm{CF}_{\text {LIM }}$ is the $(1-\alpha)$ percentile. Figure 5 a presents the evolution of the $\mathrm{CF}_{\mathrm{LIM}}$ values obtained from the referred simulation study for increasing values of $n_{1}$ (from 1 to 30), for the previously defined range of the $\mathrm{CoV}(6-20 \%$ in $2 \%$ steps), for the previously defined 
range of the concrete compressive strength (12$50 \mathrm{MPa}$ in $1 \mathrm{MPa}$ steps) and for the $90 \%$ confidence level associated to KL2. Observation of these results allows concluding that, for the case of a normal distributed strength, when the prior knowledge and the new tests data are in agreement, the required $\mathrm{CF}_{\text {LIM }}$ decreases. Considering the case where the $\mathrm{CoV}$ is $20 \%$ and the new minimum number of tests set for KL2 (i.e. $n_{1}=1$ ), the results obtained yielded a $\mathrm{CF}_{\mathrm{LIM}}$ value of 1.13 , which is lower than the one previously obtained in the absence of prior knowledge.

Given that the obtained $\mathrm{CF}_{\text {LIM }}$ value is lower than the EC8-3 proposed value (i.e. 1.2), a second simulation study was performed to produce a situation where the prior knowledge and the new test data are contradictory. The simulation study was carried out as for the previous case, but considering the prior knowledge data representing a higher concrete strength to represent a critical situation of nonconformity. It was found that, for a $\mathrm{CoV}$ of $20 \%$ and for $n_{1}=1$, the value of $X_{0}$ could not exceed the mean of the distribution of the test data by more than $15 \%$ in order to yield a $\mathrm{CF}_{\mathrm{LIM}}$ value up to 1.20 . Figure $5 \mathrm{~b}$ presents the evolution of the $\mathrm{CF}_{\mathrm{LIM}}$ values for the conditions set for the previous simulation study, but considering that $X_{0}$ exceeds the mean of the distribution of the test data by $15 \%$.

\subsection{Considering prior knowledge and KL3}

When KL3 is chosen in the context of a situation where prior knowledge exists, it is assumed that original test reports can lead to the definition of either a value of $X_{0}$, or a value of $X_{0}$ and a prior estimate of the standard deviation $s_{0}$ of the data. For the former situation (only $X_{0}$ is known), the updated estimate of the mean value $X_{\mathrm{U}}$ can also be obtained by Eq. (27). In this case, the value of $n_{0}$ reflecting the actual number of tests that were performed might be available from test reports and can be either smaller or larger than $n_{1}$. In the absence of such knowledge, an interpretation similar to the one considered for KL2 can also be applied, leading to the definition of $n_{0}$ with twice the value of $n_{1}$, in order to guarantee the level of reliability of KL3 in the absence of prior knowledge. This latter situation is considered herein to illustrate the effect of existing prior knowledge. Conclusions based on the presented results will be restricted to cases with similar conditions in terms of the $n_{0} / n_{1}$ ratio. As previously noted for the case of KL2, given the weight of the prior estimate $X_{0}$ on the value $X_{\mathrm{U}}$ (i.e. $67 \%$, given that $n_{0}$ has twice the value of $n_{1}$ ), if the information between prior and new data is contradictory, the analyst has to decide whether the prior data should or should not be taken into account.

In order to observe the effects of existing prior knowledge on the quantification of the CF for KL3, $\mathrm{CF}_{\text {LIM }}$ values were assessed using the simulation approach considered in Sect. 4.1. In order to observe the evolution of the $\mathrm{CF}_{\mathrm{LIM}}$ values, Fig. $5 \mathrm{c}$ presents the results obtained from this simulation study for increasing values of $n$ (from 1 to 30), for the previously defined range of the $\mathrm{CoV}(6-20 \%$ in $2 \%$ steps), for the previously defined range of the concrete compressive strength (12-50 MPa in $1 \mathrm{MPa}$ steps) and for the $75 \%$ confidence level associated to KL3. Observation of the results confirms that when the prior knowledge and the new tests data are in agreement, the necessary $\mathrm{CF}_{\text {LIM }}$ decreases. As can be seen in the figure, the $\mathrm{CF}_{\mathrm{LIM}}$ value corresponding to a $\mathrm{CoV}$ of $20 \%$, and to the new minimum number of tests set for KL3 (i.e. $n_{1}=1$ ) is 1.05 , which is lower than the value previously obtained in the absence of prior knowledge. It should also be noted that, since the obtained $\mathrm{CF}_{\mathrm{LIM}}$ value is larger than the EC8-3 proposed value (i.e. 1.0), there seems to be no room for a situation where the prior information and the new test data are contradictory.

For the case where both $X_{0}$ and $s_{0}$ are known, the updated estimate of the mean value $X_{\mathrm{U}}$ can now be obtained by $[45,46]$ :

$X_{\mathrm{U}}=\frac{X_{0} \cdot s_{1}^{2} / n_{1}+X_{1} \cdot s_{0}^{2} / n_{0}}{s_{0}^{2} / n_{0}+s_{1}^{2} / n_{1}}$

where $s_{1}$ is the standard deviation obtained from the new data. By considering the previously referred relation between $n_{0}$ and $n_{1}$, and by defining the variance ratio $\zeta=\left(s_{1} / s_{0}\right)^{2}$, Eq. (28) can be rearranged to yield:

$X_{\mathrm{U}}=\frac{2 \cdot X_{0} \cdot \zeta+X_{1}}{2 \cdot \zeta+1}$

By analysing Eq. (29), the following can be observed:

- When $\zeta$ takes the value of $0.5, X_{0}$ and $X_{1}$ have the same weight over the value of $X_{\mathrm{U}}$, and when $\zeta$ 

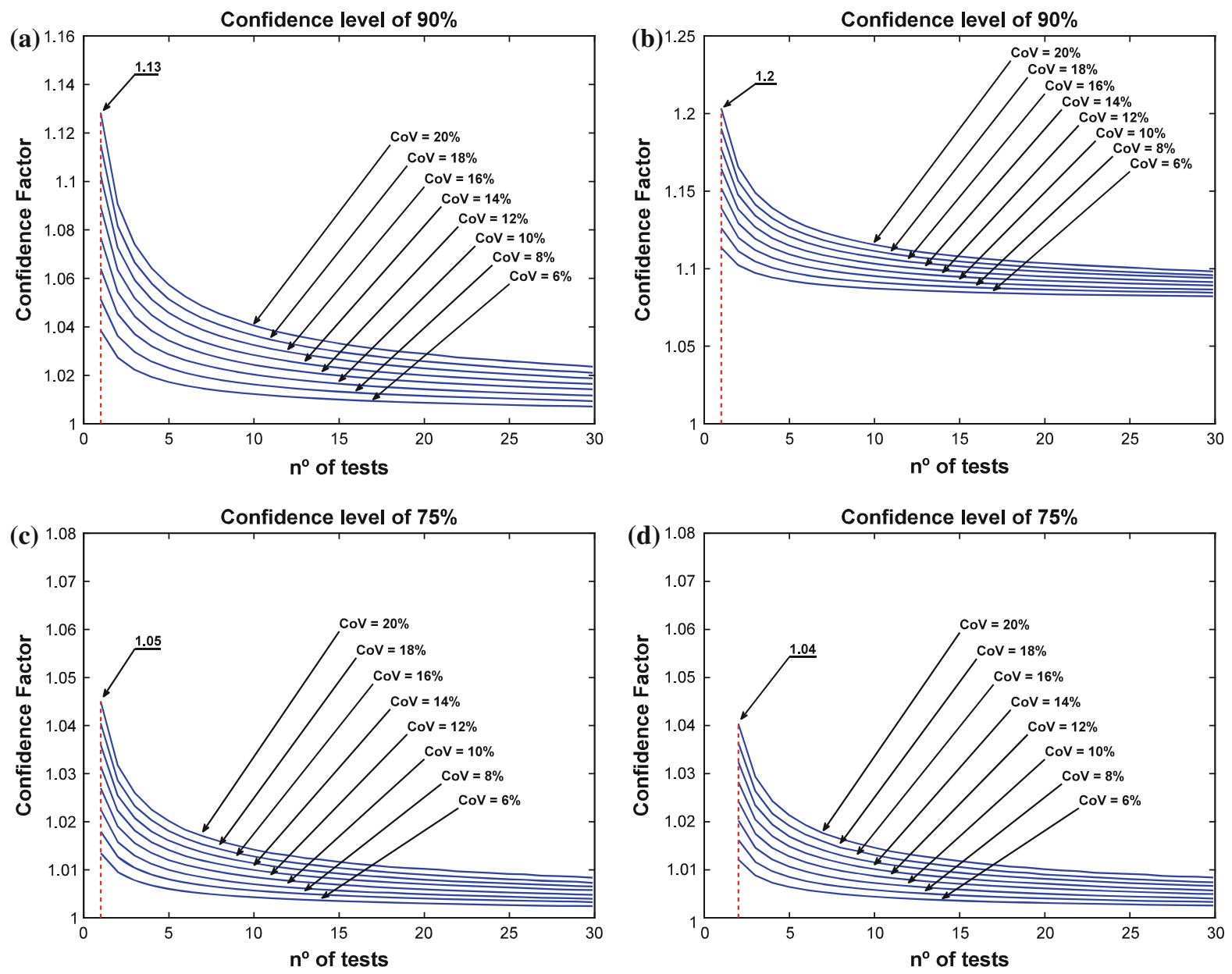

Fig. 5 Values of $\mathrm{CF}_{\text {LIM }}$ obtained by simulation considering a normal distributed strength, the existence of prior knowledge, for increasing values of $n$, for the selected range of CoVs, for KL2 (confidence level of $90 \%$ ) when the prior information and the new test data are in agreement (a), when the prior

takes the value of 1.0, Eq. (29) yields the results of Eq. (27);

- When $\zeta$ is lower than $0.5, X_{1}$ dominates the value of $X_{\mathrm{U}}$ and when $\zeta$ is larger than 0.5 , it is $X_{0}$ that dominates $X_{\mathrm{U}}$;

- As $\zeta$ increases, the value of $X_{\mathrm{U}}$ asymptotically tends to $X_{0}$.

In order to exemplify these findings and for a better interpretation of the behaviour of Eq. (29), a simulation study is presented next. It is assumed that $X_{0}$ is $35 \mathrm{MPa}, s_{0}=4.2 \mathrm{MPa}(\mathrm{CoV}=12 \%)$ and that the new test data yields values of $X_{1}$ ranging from 25 to information exceeds the mean of the new test data by $15 \%$ (b); for KL3 (confidence level of $75 \%$ ) when the prior information and the new test data are in agreement (c) and when the prior information and the new test data are in agreement and $s_{0}$ is known (d)

$45 \mathrm{MPa}$ (in steps of $1 \mathrm{MPa}$ ) and values of $s_{1}$ corresponding to CoVs ranging from 6 to $20 \%$ (in $1 \%$ steps). For each value of $X_{1}$, a range of values of $s_{1}$ is defined, in correspondence to the assumed CoVs, which then yields the corresponding values of $\zeta$. For the conditions set, the evolution of Eq. (29) is represented in Fig. 6.

In order to observe the effects of the consideration of Eq. (29) on the quantification of the CF for KL3, $\mathrm{CF}_{\text {LIM }}$ values were assessed using the previously referred simulation approach. In this case, the updated mean $X_{\mathrm{U}}$ was computed according to Eq. (29). Since 


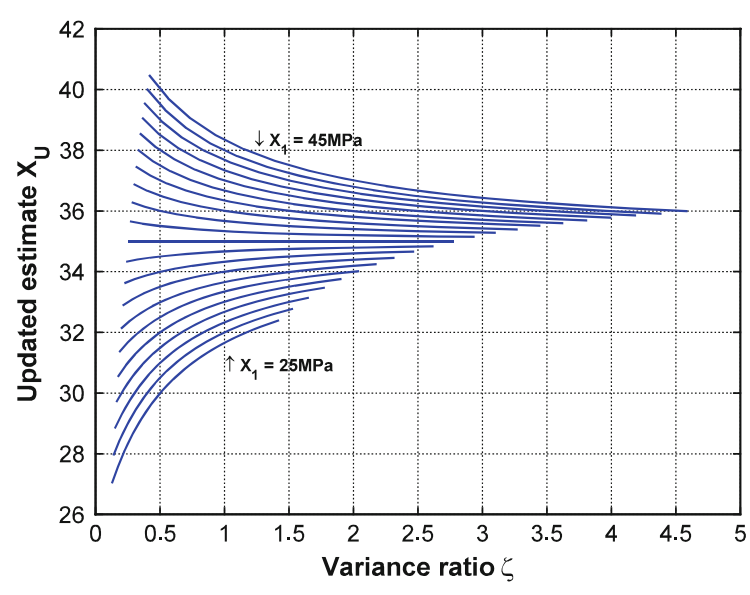

Fig. 6 Values of $X_{\mathrm{U}}$ considering the existence of prior knowledge, for the case when both $X_{0}$ and $s_{0}$ are known

Eq. (29) is based on $s_{1}$, the new minimum number of tests set for KL3 (i.e. $n_{1}=1$ ) was not able to be considered; a value of $n_{1}=2$ was used instead. In order to observe the evolution of the $\mathrm{CF}_{\mathrm{LIM}}$ values obtained in this case, Fig. $5 \mathrm{~d}$ presents the global results determined from the referred simulation study for increasing values of $n_{1}$ (from 2 to 30), for the previously defined range of the $\mathrm{CoV}$ (6-20\% in $2 \%$ steps), for the previously defined range of the concrete compressive strength (12-50 MPa in $1 \mathrm{MPa}$ steps) and for the $75 \%$ confidence level associated to KL3. Considering the case where the $\mathrm{CoV}$ is $20 \%$ and $n_{1}=2$, the results obtained yielded a $\mathrm{CF}_{\mathrm{LIM}}$ value of 1.04. To compare the performance of Eq. (27) with that of Eq. (29), the $\mathrm{CF}_{\text {LIM }}$ value obtained with Eq. (29) for $n_{1}=2$ must be determined. Observation of Fig. $5 c$ leads to a $\mathrm{CF}_{\mathrm{LIM}}$ value of 1.03 for this case. Hence, it appears that Eq. (29) leads to slightly more conservative results than Eq. (27).

\subsection{Combining data from different types of tests}

The combination of data coming from different types of tests is similar in formulation to the case of Sect. 4.2 where both $X_{0}$ and $s_{0}$ are known, Eq. (28), but without enforcing a specific relation between $n_{0}$ and $n_{1}$. Considering that $X_{0}$ and $s_{0}$ are known from core compression tests, that $X_{1}$ and $s_{1}$ are obtained from a chosen NDT and that the relation between $n_{0}$ (number of core tests) and $n_{1}$ (number of NDTs) is defined by $n_{1}=k \cdot n_{0}$, Eq. (28) can be rearranged to yield $X_{\mathrm{U}}$ given by:

$X_{\mathrm{U}}=\frac{X_{0} \cdot \zeta+k \cdot X_{1}}{\zeta+k}$

where $\zeta$ is as previously defined. In order to observe the effects of the consideration of Eq. (30) on the quantification of the $\mathrm{CF}$ for the several KLs, $\mathrm{CF}_{\mathrm{LIM}}$ values were assessed using a simulation approach similar to that of the previous Section and with the updated mean $X_{\mathrm{U}}$ computed according to Eq. (30). This simulation study considered increasing values of $n_{0}$ (from 2 to 30), three values for $k\left(2,5\right.$ and 10), $X_{0}$ values ranging from 12 to $50 \mathrm{MPa}$, in $1 \mathrm{MPa}$ steps, $s_{0}$ values corresponding to $\mathrm{CoV}$ values ranging from 6$20 \%$, in $2 \%$ steps, $X_{1}$ values defined by the mean of random samples of size $n_{1}$ drawn from a normal distribution defined by $X_{0}$ and $s_{0}$, and $s_{1}$ values defined by:

$s_{1}=\sqrt{s_{1, s}^{2}+s_{1, v}^{2}}$

where $s_{1, s}$ is the standard deviation of the sample used to obtain $X_{1}$ and $s_{1, v}$ is an additional standard deviation aiming to represent the increased variability of the NDT results. In the proposed simulation study, $s_{1, v}$ was set as $15 \%$ of $X_{0}$. According to this simulation study, parameter $\zeta$ ranges between 1.0 and 10.0. Although conclusions based on the presented results will be restricted to cases with similar conditions in terms of the $n_{0} / n_{1}$ ratio and of the considered range of $\zeta$, they allow for a qualitative interpretation of the influence of combining testing data from different test types. In order to observe the evolution of the $\mathrm{CF}_{\mathrm{LIM}}$ values obtained in this case, Figs. 7a, c, e present the results obtained from the referred simulation study for $k=2$ and for the 95, 90 and $75 \%$ confidence levels associated to KL1, KL2 and KL3, respectively, while Figs. $7 \mathrm{~b}, \mathrm{~d}, \mathrm{f}$ present similar results for $k=5$. As can be observed, results indicate that parameter $k$ has a relatively reduced influence on the $\mathrm{CF}_{\text {LIM }}$ values. For the case of $k=10, \mathrm{CF}_{\text {LIM }}$ values are slightly lower than those of $k=5$. Furthermore, it can also be seen that combining different types of tests following the considered approach leads to $\mathrm{CF}_{\text {LIM }}$ values that are lower than those obtained in the first part of the proposed study for all KLs, thus validating the previously considered hypothesis that Scenario 1 is more critical. 

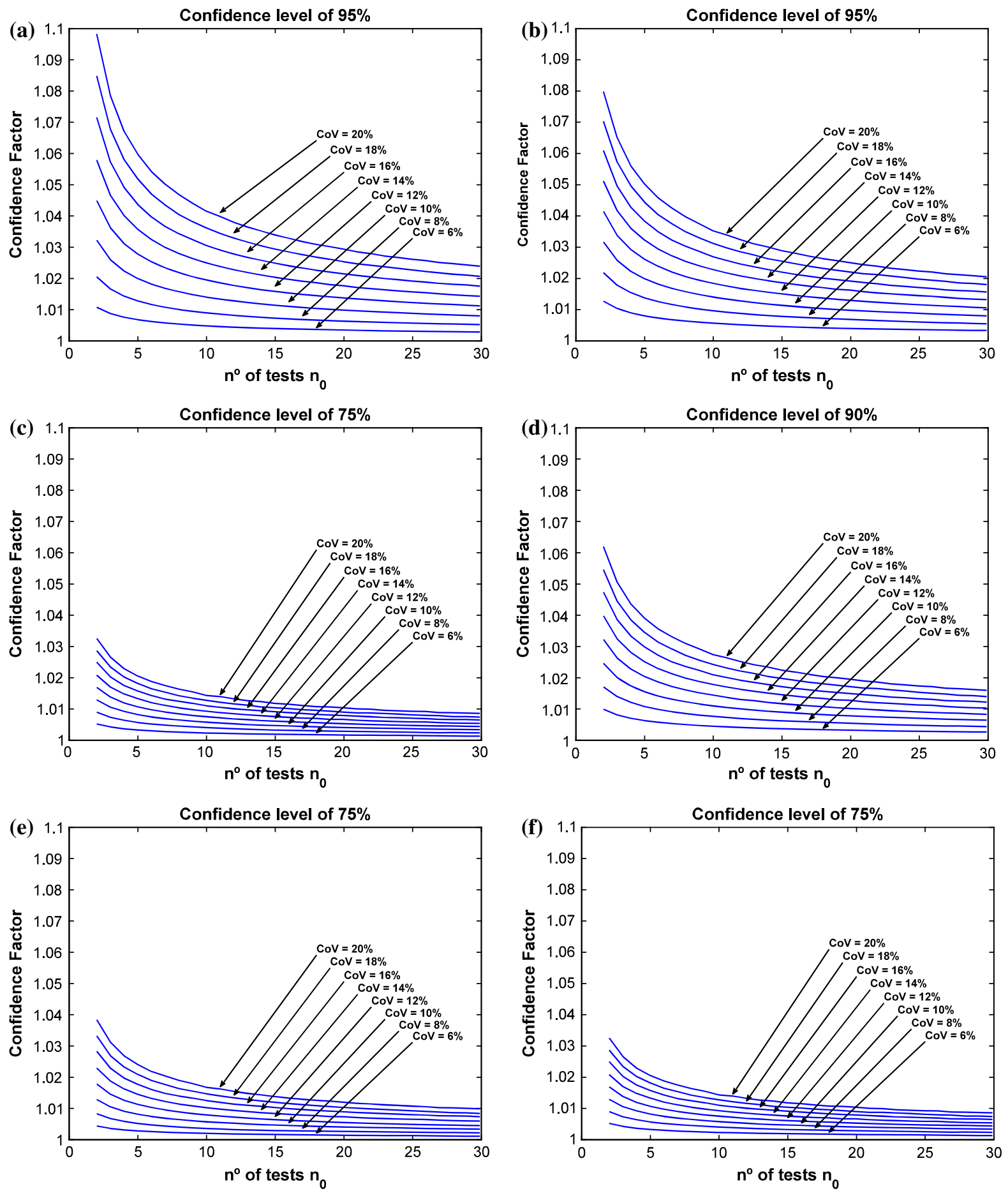

Fig. 7 Values of $\mathrm{CF}_{\text {LIM }}$ obtained by simulation considering a normal distributed strength, two sources of testing data, for increasing values of $n_{0}$, for the selected range of CoVs, for KL1

(confidence level of $95 \%$ ) and $k=2$ (a) and $k=5$ (b); for KL2 (confidence level of $90 \%$ ) and $k=2(\mathbf{c})$ and $k=5$ (d); for KL3 (confidence level of $75 \%$ ) and $k=2$ (e) and $k=5$ (f) 


\section{Conclusions}

The present study addresses the evaluation of the recommended values of the CFs proposed in the main document of the EC8-3 for the characterization of material properties of existing structures. In this context, the CF adjusts the mean estimate of a material property in order to reflect the KL that is attained in the assessment, in order to provide a design value of the property that is on the safe side. Prior to the evaluation of the CFs, the influence of the level of knowledge and of the uncertainty about the parameters entering the EC8-3 safety assessment procedure is examined. From this analysis, it is concluded that only the uncertainty related to the materials is reflected in the quantification of the EC8-3 safety measures, thus justifying the focus of the proposed study.

The reliability of the EC8-3 proposed CF values is assessed using a probabilistic framework, in which the number of material tests and the existence of prior knowledge are seen to be essential aspects for the quantification of the CFs. Although the general concept behind the CFs is independent of the type of material, the evaluation is presented for the case of $\mathrm{RC}$ structures, more specifically for the concrete compressive strength.

In the first part of the study, where the existence of prior knowledge is not considered, different underlying statistical distributions are assumed for the concrete compressive strength (normal, lognormal and Weibull distributions) and different confidence levels are associated to the quantification of the CF of each KL (95, 90 and $75 \%$ for KL1, KL2 and KL3, respectively). Moreover, the definition of the CFs reflects the critical situation that occurs when the estimate of the mean strength overestimates the real mean value. Based on the results obtained for the different distributions and assumptions of the presented study, the EC8-3 proposed CFs are seen to be more consistent with the known variance assumption. Moreover, the KL1 and KL2 CFs are believed to be adequate, assuming that the selected confidence levels are satisfactory. With respect to KL3, the EC8-3 proposed CF is not met by any of the cases studied. Nonetheless, the known variance assumption is also selected since it leads to lower values of the CF. For this case, a $\mathrm{CF}$ value of 1.08 is seen to be more adequate to the assumptions made in the study. Furthermore, considering the known variance hypothesis and for a $\mathrm{CoV}$ of $20 \%$, it was observed that if the EC8-3 minimum number of tests are met, the $\mathrm{CF}$ values could be significantly reduced for buildings higher than three-storeys (e.g. from 1.35 to 1.14, for $\mathrm{KL} 1$, and from 1.20 to 1.08 , for KL2). On the other hand, considering the unknown variance hypothesis and for a $\overline{\mathrm{CoV}}$ of $40 \%$, the EC8-3 proposed CF for KL2 and the proposed value of 1.08 for KL3 can be seen to also include such hypothesis for buildings higher than three-storeys. For the case of KL1, this situation can only be attained if the maximum $\overline{\mathrm{CoV}}$ is reduced to $32 \%$.

It was also found that, for the cases where the normal and the lognormal distribution assumptions are considered, the probabilistic framework that was defined to obtain the CF values depends only on the $\mathrm{CoV}$ of the material property under study. Hence, the proposed expressions can be applied to the properties of other materials of interest for which the referred statistical distribution assumptions are acceptable. Moreover, if the selected range of the $\mathrm{CoV}$ is acceptable for such material properties, the adequacy of the EC8-3 proposed CFs for these properties can be inferred from the results obtained for the concrete compressive strength.

In the second part of the study, the effects of prior knowledge on the quantification of the CFs are assessed. EC8-3 accounts for prior knowledge to produce a more accurate estimate of the material strength and allows the required number of tests of a given KL to be reduced. It should be noted that, in case the information between prior and new data is contradictory, the analyst has to decide whether the prior data should or should not be taken into account. By using a Bayesian framework and considering the case of a normal distributed strength, the results obtained lead to the conclusion that when the prior knowledge and the new test data are in agreement, the necessary CF decreases, when compared to the value obtained in the absence of prior knowledge. Nonetheless, the CF value obtained for KL3 is still larger than the value proposed by EC8-3. In addition, the case of combining different sources of testing data was also addressed considering the same Bayesian framework and the case of a normal distributed strength, as it is a special case of the situation where prior knowledge exists. Results of this analysis indicate that combining different types of tests leads to $\mathrm{CF}$ values that are 
lower than those obtained in the first part of the proposed study for all KLs. The presented analysis represents a possible interpretation of the code procedures but was not an extensive study of the case of existing prior knowledge. Although a qualitative evolution trend of the $\mathrm{CF}$ values was able to be observed, further research on this topic is required to be able to develop specific CF values accounting for prior knowledge.

Acknowledgments The financial support of the Portuguese Foundation for Science and Technology to the research project "Development and calibration of the Eurocode 8-Part 3 seismic safety assessment methodologies for existing buildings" (PTDC/ECM/108098/2008) is gratefully acknowledged.

\section{References}

1. Melchers RE (2001) Assessment of existing structures: approaches and research. ASCE J Struct Eng 127(4): 406-411

2. Dimitri VV, Stewart MG (2002) Safety factors for assessment of existing structures. ASCE J Struct Eng 128(2): 258-265

3. Krinitzsky EL (1993) Earthquake probability in engineering: Part 1. The use and misuse of expert opinion. Eng Geol 33(4):257-288

4. ENV 1998-3 (2005) Eurocode 8: design of structures for earthquake resistance-Part 3: Assessment and retrofitting of buildings. European Committee for Standardization

5. NTC (2008) Norme Tecniche per le Costruzioni. Decreto del Ministero delle infrastrutture, Supplemento Ordinario n.30 alla Gazzetta Ufficiale della Repubblica italiana n.29 del 4/02/2008, Italy (in Italian)

6. ASCE (2007) Seismic rehabilitation of existing buildings (ASCE/SEI 41-06). American Society of Civil Engineers, Reston, Virginia, USA

7. Franchin P, Pinto PE, Rajeev P (2008) Assessing the adequacy of a single Confidence Factor in accounting for epistemic uncertainty. In: Cosenza E, Manfredi G, Monti G (eds) Valutazione e riduzione della vulnerabilità sismica di edifici esistenti in cemento armato. Polimetrica s.a.s. International Scientific Publisher, Monza

8. Franchin P, Pinto PE, Rajeev P (2009) Confidence in the confidence factor. Convegno Finale del Progetto ReLuisDPC. Naples, Italy

9. Monti G, Alessandri S (2009) Application of Bayesian techniques to material strength evaluation and calibration of confidence factors. Convegno Finale del Progetto ReLuisDPC, Naples, Italy

10. Elefante L (2009) Dealing with uncertainties in the assessment of existing RC buildings. PhD Thesis, Università degli Studi di Napoli Federico II, Naples, Italy

11. Franchin P, Pinto PE, Rajeev P (2010) Confidence factor? J Earthq Eng 14(7):989-1007
12. Jalayer F, Elefante L, Iervolino I, Manfredi G (2011) Knowledge-based performance assessment of existing RC buildings. J Earthq Eng 15(3):362-389

13. Neville AM (1996) Properties of concrete, 4th edn. Wiley, New York

14. Bungey JH, Millard SG (1996) Testing of concrete in structures, 3rd edn. Blackie academic and professional, London, an imprint of Chapman \& Hall

15. Bartlett FM, MacGregor JG (1994) Assessment of concrete strength in existing structures. Structural Engineering Report No. 198, Department of Civil and Environmental Engineering, University of Alberta, Canada

16. ACI 228.1R-03 (2003) In-place methods to estimate concrete strength. American Concrete Institute, Farmington Hills, Michigan

17. EN 13791 (2007) Assessment of in situ compressive strength in structures and precast concrete components. European Committee for Standardization

18. Kappos AJ, Chryssanthopoulos MK, Dymiotis C (1999) Uncertainty analysis of strength and ductility of confined reinforced concrete members. Eng Struct 21(2):195-208

19. Bartlett FM, MacGregor JG (1995) Equivalent specified concrete strength from core test data. Concr Int 17(3):52-58

20. Bartlett FM (1997) Precision of in-place concrete strengths predicted using core strength correction factors obtained by weighted regression analysis. Struct Saf 19(4):397-410

21. Stewart MG (1995) Workmanship and its influence on probabilistic models of concrete compressive strength. ACI Mater J 92(4):361-372

22. Day KW (1999) Concrete mix design, quality control and specification, 2nd edn. E \& FN Spon, London

23. Tumidajski PJ, Fiore L, Khodabocus T, Lachemi M, Pari R (2006) Comparison of Weibull and normal distributions for concrete compressive strengths. Can J Civ Eng 33(10): $1287-1292$

24. Drysdale RG (1973) Variation of concrete strength in existing buildings. Mag Concr Res 25(85):201-207

25. Bartlett FM, MacGregor JG (1999) Variation of in-place concrete strength in structures. ACI Mater J 96(2):261-270

26. Wísniewski DF (2007) Safety formats for the assessment of concrete bridges: with special focus on precast concrete. PhD Thesis, University of Minho, Portugal

27. Aguiar B, Veiga V, Oliveira P (2003) Statistical analysis of compressive strength of concrete specimens. In: Proceedings of the ICPCM: A New Era of Building, Cairo, Egypt

28. Montgomery DC, Runger GC (2003) Applied statistics and probability for engineers, 3rd edn. Wiley, New York

29. Maronna RA, Martin DR, Yohai VJ (2006) Robust statistics: theory and methods. Wiley, New York

30. ISO 12491 (1997) Statistical methods for quality control of building materials and components. International Standard Organization

31. Leonardo da Vinci Pilot Project CZ/02/B/B/PP-134007 (2005) Reliability backgrounds (handbook 2), European Commission

32. Hindo KR, Bergstrom WR (1985) Statistical evaluation of the in-place compressive strength of concrete. Concr Int 7(2):44-48

33. Wong WF, Chiew SP, Ho NY (1993) Evaluation of in situ test data from existing concrete structures. In: Dhir RK, Jones MR (eds) Proceedings of the symposium concrete 
2000: economic and durable construction through excellence, vol 2. E \& FN Spon, London

34. Singh AK, Singh A, Engelhardt M (1997) The lognormal distribution in environmental applications. EPA Issue Paper EPA/600/R-97/006

35. Zhou X-H, Gao S (1997) Confidence intervals for the lognormal mean. Stat Med 16(7):783-790

36. Aoshima M, Govindarajulu Z (2002) Fixed-width confidence interval for a lognormal mean. Int J Math Math Sci 29(3):143-153

37. Krishnamoorthy K, Mathew T (2003) Inferences on the means of lognormal distributions using generalized $p$-values and generalized confidence intervals. J Stat Plan Inference 115(1):103-121

38. Shen H (2003) Nonparametric regression for problems involving lognormal distributions. PhD Thesis, University of Pennsylvania, USA

39. El-Shaarawi AH, Lin J (2007) Interval estimation for lognormal mean with applications to water quality. Environmetrics 18(1):1-10
40. Zou GY, Huo CY, Taleban J (2008) Simple confidence intervals for lognormal means and their differences with environmental applications. Environmetrics 20(2):172-180

41. Castillo E (1988) Extreme value theory in engineering. Statistical Modeling and Decision Science. Academic Press, New York

42. Lawless JF (1982) Statistical models and methods for lifetime data, 2nd edn. Wiley, New York

43. Xie M, Yang ZL, Gaudoin O (2000) More on the misspecification of the shape parameter with Weibull-to-exponential transformation. Qual Reliab Eng Int 16(4):281-290

44. Yang Z, Xie M, Wong ACM (2007) A unified confidence interval for reliability-related quantities of two-parameter Weibull distribution. J Stat Comput Simul 77(5):365-378

45. Ang AH-S, Tang WH (1984) Probability concepts in engineering: emphasis on applications to civil and environmental engineering. Wiley, New York

46. Diamantidis D (ed) (2001) Probabilistic assessment of existing structures. Joint Committee on Structural Safety. RILEM Publications S.A.R.L, Cachan 